\title{
On the Spin Structure of Magnetic Vortex Core Retrieved by Lorentz Phase Microscopy in Defocus-Calibrated TEM.
}

\author{
V.V. Volkov and Y. Zhu \\ Center for Functional Nanomaterials, Brookhaven National Laboratory, Upton, NY 11973, USA
}

Understanding the nature, in its most elementary form, is interesting because its basic principles can be applied to gain better insight into more complicated systems and smart devices. Studies of the confined, magnetic vortex state by Lorentz phase microscopy (LPM) are presented in this work. The vortex states can be experimentally defined as magnetic curl that appears at the intersection of Néel walls and in crosstie walls (known as antivortex) and are often observed at meso- and nanoscale in patterned arrays of magnetic elements. Qualitative experiments by magnetic force microscopy (MFM) [1] and, recently, by spin-polarized scanning tunneling microscopy (SP-STM) [2] have shown that vortices are 3D-structures that possess nanometer-sized cores in which the curling magnetization turns out-of-plane, avoiding the high energetic cost of anti-aligned moments. Another intriguing question is how the chirality of vortices is taking control over magnetization dynamics [3]. Notice that experimental data in $[1,2]$ should be treated with care because of possible artifacts caused by the interaction of magnetic tip at sample surface. In contrast, the electron probe used in transmission mode by LPM is free from such tip-surface artifacts, and hence, is potentially useful for retrieving direct information on the core spin structure of magnetic vortices averaged over the film thickness. Notice that LPM [4,5] is a complementary technique to the well-established off-axis electron holography; however, due to different requirements to electron optics - coherence of the beam source and experimental setup - their performance can be better optimized on different length scales. In addition, quantitative phase retrieval by LPM requires calibration of image defocus in electron microscope for symmetrically defocused Fresnel images (Fig. 1) usually recorded with the TEM objective lens (OL) off.

In current work by LPM analysis of the projected magnetic induction in close proximity to magnetic vortices and antivortices we provide an experimental evidence for the out-of-plane 3D-spin structure of magnetic vortex core. For this purpose a magnetic array of $6 \times 6 \mu \mathrm{m}^{2}$ square islands was analyzed. It was made of $40 \mathrm{~nm}$ thick Co-films grown on $50 \mathrm{~nm}$ thick silicon-nitride membrane by means of electron-beam evaporation through a shadow mask in a UHV chamber. Co-films with grain size 8-10 $\mathrm{nm}$ consisting of hcp/fcc mixture of magnetic phases were also examined by the conventional TEM using a $300 \mathrm{keV}$ microscope JEM3000F. Defocus calibration of the same microscope used for LPM imaging in Lorentz mode (with OL turned off) was controlled by the objective mini-lens (OM) current at medium magnifications $(M=1,000-10,000 x)$. A calibration curve for measured "effective" OM defocus (def, "nm") versus real z-shift $(\mathrm{z}, \mu \mathrm{m})$ of the sample image plane is shown in Fig. 1. As expected, the defocus calibration curve is independent of the magnifications used and obeys a simple linear law $Z(\mu \mathrm{m})=-5.98^{*} \operatorname{def}$ ("nm"). Quantitative phase-gradient map used for in-plane magnetization mapping $\left(\mathrm{M}_{\mathrm{x}}, \mathrm{M}_{\mathrm{y}}\right)$ shown in Fig. 2 have been reconstructed with known image defocus (Fig. 1) using the LPM phase retrieval process from [4,5]. Using a commonly accepted assumption that modulus of total magnetization $\bmod (\mathrm{M})$ over the exchange length compatible with the film grain size remains constant, we get a good approximation for the $\mathrm{M}_{\mathrm{z}}$ spin component as $\bmod \left(\mathrm{M}_{\mathrm{z}}\right)=\operatorname{sqrt}\left(\mathrm{M}^{2}-\mathrm{M}_{\mathrm{y}}^{2}-\mathrm{M}_{\mathrm{x}}^{2}\right)$. Such function as measure for the out-of-plane spin component is plotted by line scan across the vortex core in Fig. 3, which agrees well with data obtained so far only with surface-probe methods by MFM [1] and SP-STM [2]. Experimental procedures on how to establish the vortex chirality will also be presented. 


\section{References:}

[1] T. Shinjo, T. Okuno, R. Hassdorf, K. Shigeto, T. Ono, Science 289, 930 (2000).

[2] A. Wachowiak et al., Science 298, 577 (2002).

[3] S.-B. Choe et al., Science 304, 420 (2004)

[4] V.V. Volkov and Y. Zhu, Phys. Rev. Lett., 91 (2003) 043904.

[5] V.V. Volkov and Y. Zhu, Ultramicroscopy, 98 (2004) 271.

[6] Work was supported by US DOE, DE-AC02-98CH10886.

The authors acknowledge M. Malac for providing patterned magnetic Co sample.
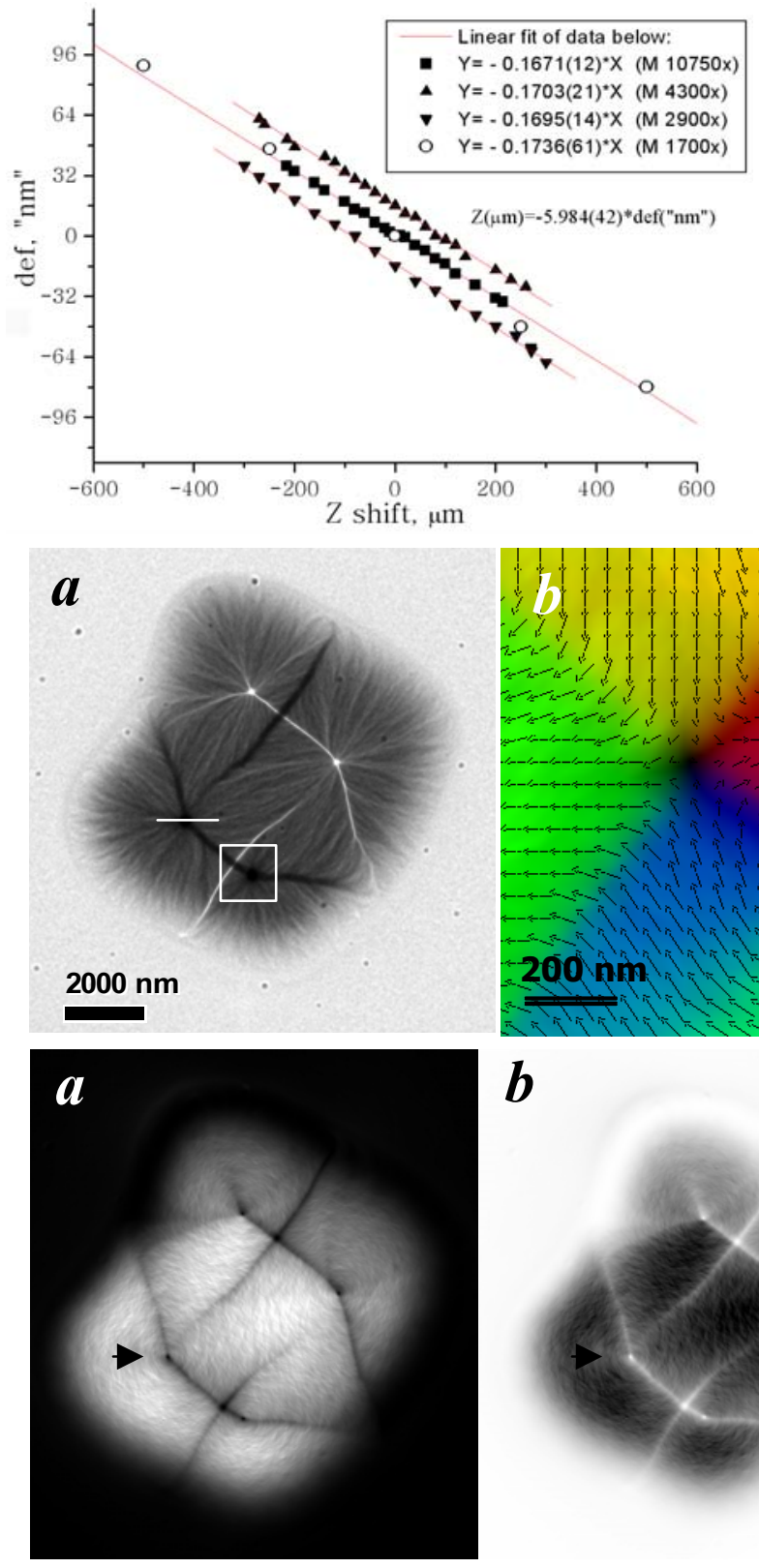
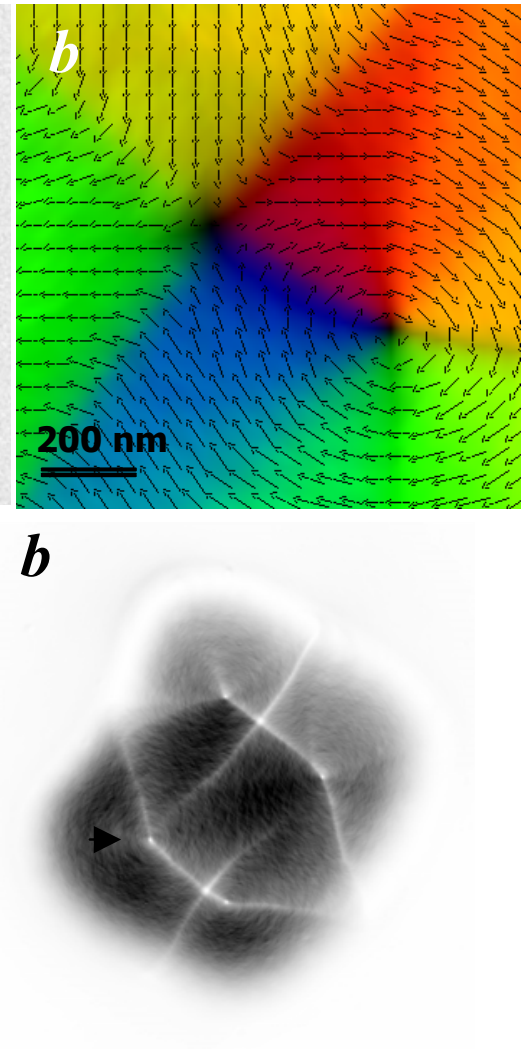

Fig.1 Calibration curves for the measured "effective" image defocus versus real image $\mathrm{z}$-shift controlled by the OM current in JEM3000F microscope operating in Lorentz mode for medium magnifications (M) at 300 $\mathrm{keV}$. For clarity, data collected at different $\mathrm{M}$ are shifted along the defaxis.

Fig 2. Defocused Fresnel image of the Co-element (a) and projected magnetization map (b) reconstructed by LPM. The vector map of in-plane spin component in vicinity of vortex and antivortex cores (b) correspond to $10 \mathrm{x}$-magnified boxed area of the experimental image (a).

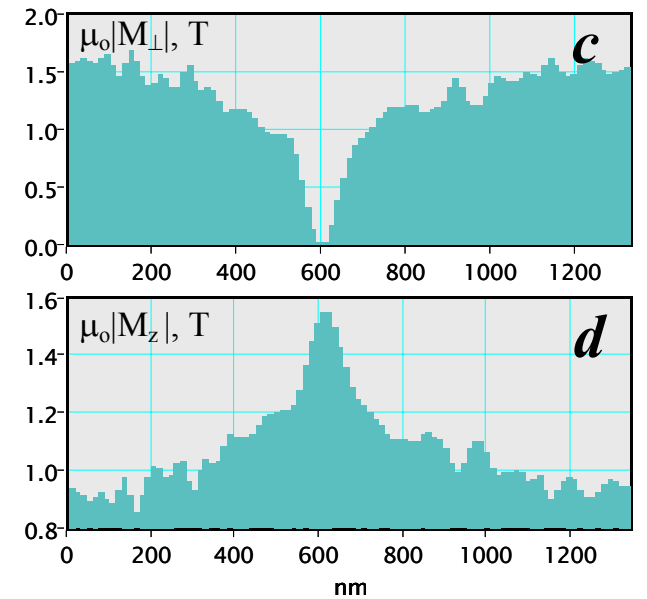

Fig. 3 Modulus of the projected in-plane magnetization (a) and complementary out-of-plane magnetization (b) with corresponding line profiles (c) and (d) across the same vortex core pointed by black arrows as shown by the white-line bar in the experimental image of Fig. 2 (a). The FWHMwidth of the vortex core with out-of-plane spin rotation along the line scan (c, d) is about $80 \mathrm{~nm}$. 